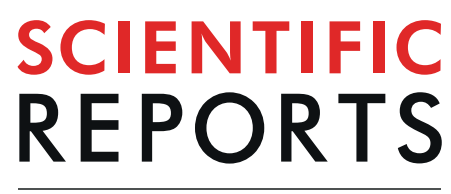

natureresearch

Check for updates

\title{
Alterations to neuromuscular properties of skeletal muscle are temporally dissociated from the oxygen uptake slow component
}

Trishan Gajanand ${ }^{1,5,6}$, Sonia Conde Alonso ${ }^{2,6 凶}$, Joyce S. Ramos ${ }^{3}$, Jean-Philippe Antonietti ${ }^{4}$ \& Fabio Borrani²

To assess if the alteration of neuromuscular properties of knee extensors muscles during heavy exercise co-vary with the $\mathrm{SCV}\left(\mathrm{VO}_{2}\right.$ slow component), eleven healthy male participants completed an incremental ramp test to exhaustion and five constant heavy intensity cycling bouts of 2, 6, 10, 20 and 30 minutes. Neuromuscular testing of the knee extensor muscles were completed before and after exercise. Results showed a significant decline in maximal voluntary contraction (MVC) torque only after 30 minutes of exercise $(-17.01 \% \pm 13.09 \% ; p<0.05)$ while single twitch (PT), $10 \mathrm{~Hz}(\mathrm{P} 10)$, and $100 \mathrm{~Hz}$ (P100) doublet peak torque amplitudes were reduced after 20 and 30 minutes $(p<0.05)$. Voluntary activation (VA) and $\mathrm{M}$-wave were not affected by exercise, but significant correlation was found between the SCV and PT, MVC, VA, P10, P100, and P10/P100 ratio, respectively $(p<0.015)$. Therefore, because the development of the SCV occurred mainly between 2-10 minutes, during which neuromuscular properties were relatively stable, and because PT, P10 and P100 were significantly reduced only after 20-30 minutes of exercise while $\mathrm{SCV}$ is stable, a temporal relationship between them does not appear to exist. These results suggest that the development of fatigue due to alterations of neuromuscular properties is not an essential requirement to elicit the SCV.

At the onset of constant power exercise, the muscles requirements for ATP re-synthesis increase immediately following exercise onset. The same cannot be said about the oxygen uptake $\left(\dot{\mathrm{V}} \mathrm{O}_{2}\right)$ response that instead, displays a sluggishness to fully activate metabolism ${ }^{1-3}$. During exercise below the lactate threshold, $\dot{\mathrm{V}} \mathrm{O}_{2}$ rises mono-exponentially to a new steady-state ${ }^{3,4}$ and from unload pedalling, the rise of $\dot{\mathrm{V}}_{2}$ increases as a linear function of work-rate ${ }^{5}$. However, during constant-load exercise completed at intensities above the lactate threshold, the $\dot{\mathrm{V}} \mathrm{O}_{2}$ response becomes more complex with a second rise in $\dot{\mathrm{VO}}_{2}$, developing slowly, which is superimposed onto the initial $\dot{\mathrm{VO}}_{2}$ response ${ }^{6}$. This slowly developing rise in $\dot{\mathrm{VO}}_{2}$, termed the slow component of $\dot{\mathrm{V}} \mathrm{O}_{2}(\mathrm{SCV})$, results in a greater end-exercise $\dot{\mathrm{VO}}_{2}$ than that predicted by the sub-LT $\dot{\mathrm{V}} \mathrm{O}_{2}$-power output relationship. It has been proposed that the inefficiency which leads to the SCV originates primarily from the active muscles ${ }^{7}$. However, the reason for this observed inefficiency in the muscle is not clear and may potentially result from reduction of ATP production per mole of oxygen (P/O ratio), diminution of the energy yield per unit of hydrolysed ATP, alteration of neuromuscular properties of muscle filament to produce force, and/or deterioration of the motor pattern of the motion ${ }^{8}$. However, the potential link between the alteration of neuromuscular properties of muscle filament and progressive muscle inefficiency, and therefore the SCV, is not well explored. The capability of muscle to produce force progressively declines during high-intensity exercise when fatigue gradually develops ${ }^{9}$. It is widely accepted that alterations of the metabolic milieu of locomotor muscles are mainly responsible for the

${ }^{1}$ Department of Exercise Sciences, Faculty of Science, University of Auckland, Auckland, New Zealand. ${ }^{2}$ Institute of Sport Sciences of University of Lausanne (ISSUL), Faculty of Biology and Medicine, University of Lausanne, Lausanne, Switzerland. ${ }^{3}$ SHAPE Research Centre, Exercise Science and Clinical Exercise Physiology, College of Nursing and Health Sciences, Flinders University, Bedford Park, South Australia, 5042, Australia. ${ }^{4}$ Institute of Psychology, University of Lausanne, Lausanne, Switzerland. ${ }^{5}$ School of Human Movement and Nutrition Sciences, The University of Queensland, St Lucia, Queensland, Australia. ${ }^{6}$ These authors contributed equally: Trishan Gajanand and Sonia Conde Alonso. ${ }^{凶}$ e-mail: sonia.condealonso@unil.ch 


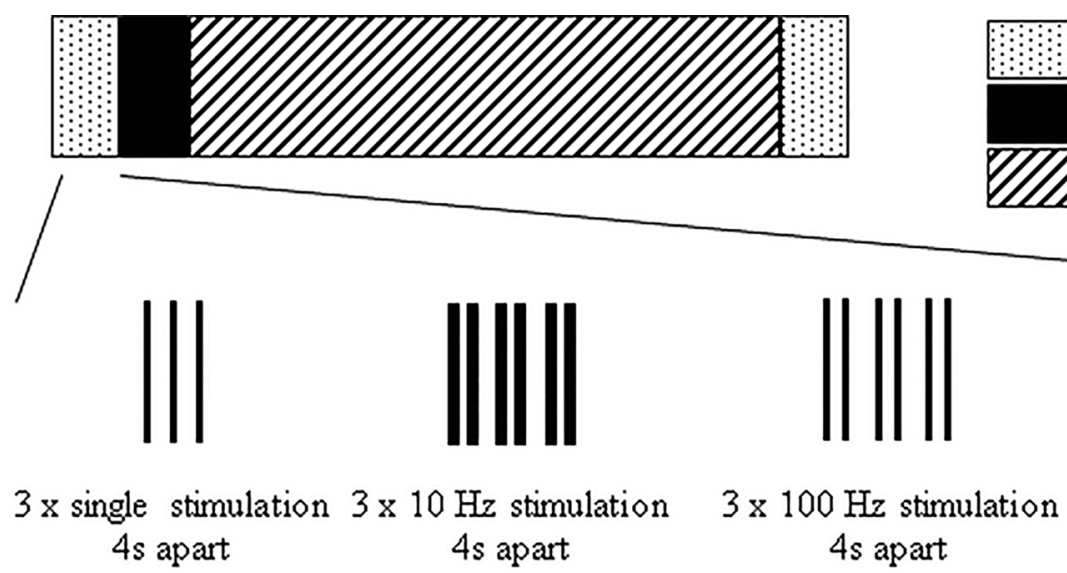

Neuromuscular testing

Rest period

Exercise

Figure 1. Description of events completed during experimental testing (A) and during neuromuscular testing (B). Neuromuscular tests (dotted box) were completed prior to the rest period (filled box) and after exercise (box with diagonal lines). Neuromuscular testing involved three single stimulations (single solid lines) followed by three stimulations at $10 \mathrm{~Hz}$ (thick-double solid lines) and then three stimulations at $100 \mathrm{~Hz}$ (thin-double solid lines). Each stimulation had a four second separation. Finally, three MVCs were completed with superimposed $100 \mathrm{~Hz}$ doublets applied (empty box with thin-double solid lines), each separated by a minute rest period.

decline in force. Indeed, neuromuscular properties of knee extensor muscles are sensitive to the accumulation of muscle metabolites such as adenosine diphosphate (ADP), inorganic phosphate $\left(\mathrm{P}_{\mathrm{i}}\right)$, hydrogen ion $\left(\mathrm{H}^{+}\right)$, and magnesium ion $\left(\mathrm{Mg}^{2+}\right)$. Muscular force production is reduced by increases in $\left[\mathrm{P}_{\mathrm{i}}\right],\left[\mathrm{Mg}^{2+}\right]$, and $\left[\mathrm{H}^{+}\right]$while augmented by an increase in $[\mathrm{ADP}]^{10}$. Additionally, increased $[\mathrm{ADP}]$ reduces cross-bridge cycling rate ${ }^{10}$.

Since the SCV occurs during high-intensity exercise, and because high-intensity exercise is always associated with changes in metabolite concentration that may produce an alteration in neuromuscular properties of muscle filament, the latter may be considered a putative mediator of the SCV (in line with current views; ${ }^{11-14}$ ).

Standardised investigative methods of neuromuscular function, such as peripheral nerve stimulation (PNS), have been extensively used to explore the complex relationship between exercise and fatigue. For instance, using PNS, Decorte and colleagues showed that during exhaustive constant-load cycling at $80 \%$ of maximum aerobic power output, neuromuscular properties were significantly reduced as early as $20 \%$ of the total duration of cycling, indicating a potential link with the $\mathrm{SCV}^{15}$. Although, little is known about the possible relationship between the SCV and the alteration of neuromuscular properties of knee extensors, Keir and colleagues ${ }^{16}$ in 2016 showed a significant association between the decrements in muscle torque and the SCV, without changes in muscle activation over the course of the exercise. Also in an in vivo study using cycle ergometry, Cannon and colleagues have shown that changes in velocity-specific peak power generated in the initial minutes of exercise were correlated to the SCV measured between three and eight minutes of heavy and severe exercise ${ }^{17}$. Results from the same working group suggest that the SCV during heavy exercise arises from both contractile and mitochondrial sources ${ }^{17}$. Furthermore, using self-paced dynamic concentric extension/flexion of the knee and interleaving voluntary and electrically evoked contractions, Froyd and colleagues have shown, even without measuring directly $\mathrm{VO}_{2}$ kinetics, that fatigue progresses with similar dynamics to those expected of the SCV during an approximately 6-min time trial ${ }^{18}$. However, these findings do not show the mechanism linking the alteration of neuromuscular properties of knee extensors, per se, and the SCV.

The aim of the present study was to quantify the alteration of neuromuscular properties of knee extensors during heavy exercise and to see if these impairments co-vary, as function of time, with the SCV amplitude. The hypothesis was that the SCV amplitude correlates with the change in neuromuscular properties of knee extensor muscles, depicted by a decrease in evoked peak torque.

\section{Methods}

Participants. Eleven healthy, recreationally active, male participants (mean $\pm S D$, age $27 \pm 6.6$ years, body mass $76 \pm 7.6 \mathrm{~kg}$, and height $179 \pm 8.1 \mathrm{~cm}$ ) were recruited for this study. The participants were provided with a participant information sheet outlining the procedures involved, time commitment, and requirements of the study. Participants were screened using a self-administrated pre-exercise health questionnaire designed to identify those who may be at risk of an adverse event during exercise. Participants were advised of their right to withdraw from the study at any time without disadvantage.

Participants were asked to avoid, in the $24 \mathrm{~h}$ preceding a testing session, strenuous physical activity, alcohol, tobacco, and caffeine. Furthermore, participants were asked not to consume any food for the $3 \mathrm{~h}$ preceding a test and to arrive fully hydrated. All tests were completed at a similar time of day $( \pm 1 \mathrm{~h})$. The study was approved by the local humans Ethics Committee and conformed to the latest revision (2013) of the Declaration of Helsinki. All participants provided written informed consent prior to participation.

Experimental design. This study involved each participant attending six separate laboratory sessions, with at least a $48 \mathrm{~h}$ interval between tests, over a three-week period. All tests were completed in an air-conditioned 
$\left(21^{\circ} \mathrm{C} \pm 1{ }^{\circ} \mathrm{C}\right)$ exercise physiology laboratory. The first session involved an incremental ramp test on a cycle ergometer (Velotron, RacerMate, Seattle, WA, USA). This test was used to assign a work-rate for the subsequent five experimental sessions during which constant work-rate exercise was completed. Following the incremental ramp test, participants were familiarised with the procedure to be used to evaluate neuromuscular function. The five experimental sessions (Fig. 1A) involved participants cycling for different durations of time in a random order at an identical power (heavy domain, see below). Neuromuscular evaluation was performed before exercise, and within 1-minute of completing constant work-rate exercise. This was completed to determine the central and peripheral fatigue through neural and neuromuscular properties of the knee extensor muscles.

Testing procedures. Incremental ramp test. Incremental ramp exercise test was completed in order to determine the gas exchange threshold (GET) and peak oxygen consumption $\left(\dot{\mathrm{V}} \mathrm{O}_{\text {2peak }}\right)$. After a three-minute rest period seated on the cycle ergometer, participants performed six minutes of baseline cycling at 60 watts, after which, the work rate was increased by the rate of 30 watts each minute until reaching the limit of tolerance. The ergometer allows participants to cycle at a constant power output independent of pedal rate, though participants were asked to maintain a pedal rate of 85 revolutions per minute (rpm). Verbal encouragement was provided throughout the test. The test was terminated when the pedal rate dropped by more than $10 \mathrm{rpm}$ (i.e. $75 \mathrm{rpm}$ ). All cycle tests were completed on an electromagnetically braked cycle ergometer where the seat and handlebars were fully adjustable both vertically and horizontally. The horizontal and vertical direction of both the seat and handlebars were adjusted to suit each participant and were recorded following the ramp test and replicated for subsequent tests. Pulmonary gas exchange and ventilation were measured from the beginning of the rest period until cessation of the test.

Step transition tests. Each participant attended a total of five experimental sessions during which cycling at a constant-load were completed. The test began with a 5 -minute rest period before participants completed three minutes of unloaded cycling ( 20 watts). At the end of the three minutes, an immediate transition to the work rate equal to $30 \% \Delta$ (GET plus $30 \%$ of the difference between the work rate at the GET and $\mathrm{V}_{2 \text { peak }}$; heavy exercise) was imposed with the duration altered at each session $(2,6,10,20,30$ minutes). Constant power was maintained at $85 \mathrm{rpm}$ and was maintained for the duration specified for each of the tests.

Neuromuscular evaluation. Neuromuscular evaluation (Fig. 1B) consisted of (1) 3 x single supra maximal electrical stimulations, each separated by four seconds, (2) $3 \mathrm{x}$ paired at $10 \mathrm{~Hz}$ (two stimulation pulses separated by $100 \mathrm{~ms}$ ) and $3 \mathrm{x}$ paired at $100 \mathrm{~Hz}$ (two stimulation pulses separated by $10 \mathrm{~ms}$ ) electrical stimulations, each separated by four seconds, and (3) $3 \times$ five-second isometric maximal voluntary contraction (MVC) tests of the knee extensor muscles during which a $100 \mathrm{~Hz}$ doublet was superimposed to the MVC. A one-minute rest period separated each MVC. Strong, standardised, verbal encouragement was provided throughout the MVC. In order to increase the contact between the electrode and the skin during all electrical stimulations, a pressure was applied to the cathode electrode using a wooden handle with a rubber end. Note that during post exercise, each sequence was repeated only one time in order to diminish the possible effect of recovery time. Less than one minute was required to position the participant for testing after exercise.

Measurements. Pulmonary gas exchange. During all tests, pulmonary gas exchange was continuously measured using a computerised system (MetaMax 3b, Cortex, Leipzig, Germany). The system used an infrared sensor and an electrochemical cell to measure fractional concentrations of $\mathrm{CO}_{2}$ and $\mathrm{O}_{2}$ in expired gas. A digital transducer turbine assessed inspired and expired gas volume. A capillary line was used to continuously sample gas concentration. The transducer and the capillary line were securely attached to the facemask, which was firmly fitted to the participants face using Velcro straps. Immediately before each exercise test, the gas analysers were calibrated with gases of known concentration $\left(\mathrm{O}_{2}=14.01 \%, \mathrm{CO}_{2}=6.03 \%\right)$, and the turbine volume transducer was calibrated using a three-litre Rudolph syringe (Cortex, Leipzig, Germany).

$\dot{\mathrm{V}} \mathrm{O}_{2 \text { peak }}$ was noted as the highest 30 -second average value attained during the incremental ramp test. The GET was determined using a number of measurements: (1) visual examination for the first disproportionate increase in $\mathrm{CO}_{2}$ production $\left(\dot{\mathrm{V}} \mathrm{CO}_{2}\right)$ from $\dot{\mathrm{V}} \mathrm{CO}_{2}$ versus $\dot{\mathrm{V}} \mathrm{O}_{2}$ graph, (2) an increase in ventilatory equivalent of oxygen $\left(\dot{\mathrm{V}}_{\mathrm{E}} / \dot{\mathrm{VO}}_{2}\right)$ without increase in ventilatory equivalent of carbon dioxide $\left(\dot{\mathrm{V}}_{\mathrm{E}} / \dot{\mathrm{V}} \mathrm{CO}_{2}\right)$, and (3) increase in partial pressure of end-tidal oxygen with no decrease in partial pressure of end-tidal carbon dioxide. Subsequently, the work rate that would require $30 \% \Delta$ was calculated and assigned for the experimental tests after accounting for the mean response time for $\dot{\mathrm{V}} \mathrm{O}_{2}$ during ramp exercise $(2 / 3$ of the ramp rate was subtracted from the work rate at the gas exchange threshold and $\dot{\mathrm{VO}}_{2 \text { peak }}$, i.e. 20 watts $)^{19,20}$.

PNS. Electrical stimulation was delivered using a high-voltage stimulator (model DS7, Digitimer Stimulator, Hertfordshire, UK). Low intensity stimulation $(\sim 20 \mathrm{~mA})$ was used to locate the femoral nerve by means of a cathode ball electrode $(0.5 \mathrm{~cm}$ diameter $)$ which was manually pressed into the femoral triangle and maneuvered until the femoral nerve was properly located (determined by observing contraction of the leg). A $5 \mathrm{~cm}$ diameter cathode electrode (American Imex, CA, USA) was then placed on the site after it was cleaned with an alcohol wipe. The anode, a rectangular electrode $(18 \times 7 \mathrm{~cm}$, American Imex, CA, USA), was placed opposite the cathode in the gluteal fold. Both the cathode and anode electrodes were worn during exercise and therefore both were taped to the skin using micropore tape (3 M Micropore, St. Paul, MN, USA) to limit movement. To determine maximal stimulation, single electrical stimulations (rectangular pulse, $1 \mathrm{~ms}$ duration, $400 \mathrm{~V}$ ) were delivered to the nerve and progressively increased until a plateau in the twitch torque and $\mathrm{M}$-wave amplitude were achieved. The current that achieved plateau was increased by $20 \%$, which was then used for subsequent tests. 
Torque measurement. The evaluation of neuromuscular function was conducted on the right knee extensor muscles with participants seated in a Biodex isokinetic dynamometer (Biodex Medical Systems Inc., Shirley, NY, USA). The hip and knee angles were fixed at $90^{\circ}\left(0^{\circ}=\right.$ full knee extension $)$ with the ankle strapped to the lever arm of the Biodex. The rotational axis of the dynamometer was aligned with the lateral epicondyle of the femur, found after palpation. Two crossover straps were placed firmly across the shoulders to limit upper body movement and one strap was placed midway across the thigh of the right leg. Participants were asked to cross their arms across their chest during testing. Adjustments made to the seat position and to the lever arm of the Biodex were recorded for each participant during familiarisation and reproduced for subsequent tests.

Electromyography recordings. Once participants were seated, the right vastus medialis (VM) and vastus lateralis (VL) muscles were palpated and prepared for electromyogram signal (EMG) recording. To reduce impedance, the skin around the belly of the muscles was shaven, lightly abraded (3 M Red Dot Trace Prep, Ontario, Canada) and cleaned using 70\% isopropyl alcohol wipes (Kendall Company, Mansfield, MA, USA). One pair of silver-chloride electrodes ( $3 \mathrm{M}$ Red Dot, St. Paul, MN, USA) of $10 \mathrm{~mm}$ diameter with an interelectrode (center to center) distance of $2 \mathrm{~cm}$ were then placed lengthwise over the prepared muscle. The ground electrode was placed over the patella of the right leg. EMG and torque signals were recorded through chart software (v. 5.5.6, ADInstruments, Sydney, Australia). EMG signals were amplified with a bandwidth frequency ranging from $1.5 \mathrm{~Hz}$ to $2 \mathrm{kHz}$ (common mode rejection $=90 \mathrm{~dB}$; impedance $=100 \mathrm{M} \Omega$; gain $=1000$ ). The myoelectric and mechanical responses were digitised on-line at a sampling frequency of $2000 \mathrm{~Hz}$ and stored for off-line analysis.

Data analysis. Oxygen uptake kinetic analysis. The breath-by-breath $\dot{\mathrm{V}}_{2}$ data from each of the $30 \% \Delta$ tests were initially examined to exclude errant breaths caused by coughing, swallowing, sighing, etc., and those values lying more than three standard deviations from the model $\mathrm{V}_{2}$ were considered outliers and were removed. The breath-by-breath data from the different exercise durations were subsequently linearly interpolated to provide second-by-second values, and, for each individual, repetitions from different durations were time aligned to the start of exercise and the ensemble averaged. The primary component (phase 2) kinetics were isolated to identify the mono-exponential region and modelled by the following equation:

$$
\dot{V} O_{2}(t)=\dot{V} O_{2 b}+A_{p} \cdot\left(1-\exp { }^{\left(-\frac{t-t d_{p}}{\tau_{p}}\right)}\right) \cdot U
$$

Where $\dot{\mathrm{VO}}_{2}(\mathrm{t})$ represents $\dot{\mathrm{V}} \mathrm{O}_{2}$ at a given time $\mathrm{t} ; \mathrm{U}=0$ for $\mathrm{t}<\mathrm{td}_{1}$ and $\mathrm{U}=1$ for $\mathrm{t} \geq \mathrm{td}_{1}$

$\dot{\mathrm{V}} \mathrm{O}_{2 \mathrm{~b}}$ is the $\dot{\mathrm{V}} \mathrm{O}_{2}$ during unloaded cycling defined as the mean $\dot{\mathrm{V}} \mathrm{O}_{2}$ measured over the final 90 seconds of baseline pedaling; $A_{P}$ is the asymptotic amplitudes for the primary phases; $\tau_{p}$ is the time constant, and $t_{p}$ represents the time delay. Since the focus of this study was the SC, the cardiodynamic phase was removed from analy$\operatorname{sis}^{21,22}$, and therefore, not modelled, in order to ensure that the early initial component did not influence the results ${ }^{23}$. Initially, the fitting window extended from 20 seconds (i.e., at the end of phase I) to 80 seconds (only $60 \mathrm{~s}$ into the exercise). The window was lengthened iteratively in order to attain four series of the initial window length. For each window length, the parameters of the model were determined with an iterative procedure by minimising the sum of the mean squares of the differences between the model $\dot{\mathrm{VO}}_{2}$ and actual $\dot{\mathrm{VO}}_{2}$.

Identification of the end of the primary phase was completed using H.B. Rossiter criteria consideration ${ }^{24,25}$.

As such, the amplitude of the slow component at time 2, 6, 10,20, and 30 minutes were assigned the value $\left(A_{\mathrm{SX}}\right)$ and were defined as the difference between the value of $\dot{\mathrm{VO}}_{2}$ at a given time and the sum of the primary phase and the $\dot{\mathrm{V}}_{2 \mathrm{~b}}$ at the same given time.

SCV was also described as a percentage of the primary component (SCV\%) since this ratio would provide information regarding the loss of efficiency.

Neuromuscular function analysis. From the EMG trace of single stimulations, peak-to-peak amplitude (M-waves) of the VL (MWVL) and VM (MWVM) were measured. Peak torque (PT) was determined from the torque signal of the single twitch. The highest torque achieved during MVC in their respective conditions were taken as the MVC torque. The PT of doublet stimulations were quantified and termed P10 and P100 for $10 \mathrm{~Hz}$, and $100 \mathrm{~Hz}$, respectively. In addition, the P10-to-P100 ratio (P10/P100) was calculated to assess for the occurrence of low or high frequency fatigue.

The voluntary activation (VA) level was calculated by expressing any increment in torque evoked during maximal isometric contractions (superimposed twitch) as a fraction of the amplitude of the response evoked by the potentiated doublet ${ }^{26}$.

In agreement with the work by Strojnick and colleagues, the following correction factor (CF, the ratio between the torque just before the superimposed doublet divided by MVC peak torque) was used in order to take into account the possibility that the superimposed twitch was not necessarily applied when the torque level was at the true maximal voluntary force ${ }^{27}$.

$$
V A=100-\left[C F \cdot\left(\frac{\text { superimposed doublet }}{\text { potentiated doublet }}\right)\right] \cdot 100
$$

All data presented are the average of three measurements in pre, and a single measurement on post. 


\begin{tabular}{|l|l|l|}
\hline & Amplitude $\left(\mathbf{l} \mathbf{m i n}^{-1}\right)$ & Amplitude (\% of Ap) \\
\hline $\mathrm{AS}_{2}$ & $0.037 \pm 0.056$ & $1.9 \pm 2.5$ \\
\hline $\mathrm{AS}_{6}$ & $0.298 \pm 0.130^{*}$ & $16.6 \pm 8.6^{*}$ \\
\hline $\mathrm{AS}_{10}$ & $0.373 \pm 0.150^{*}$ & $20.9 \pm 10.2 *$ \\
\hline $\mathrm{AS}_{20}$ & $0.450 \pm 0.202 * \&$ & $25.3 \pm 13.2 * \&$ \\
\hline $\mathrm{AS}_{30}$ & $0.452 \pm 0.246 * \&$ & $29.1 \pm 16.3 * \& \%$ \\
\hline
\end{tabular}

Table 1. Time course of slow component amplitude in absolute, and in percentage of the primary component. AS2, AS6, AS10, AS20, and AS30 are the amplitude of the slow component at time 2, 6, 10, 20, 30 min respectively. Ap is the amplitude of primary component. *Significantly different from $2 \mathrm{~min}$. \&: Significantly different from $6 \mathrm{~min}$. \%: Significantly different from $10 \mathrm{~min}$. Data are presented as mean \pm SD.

\begin{tabular}{|l|l|l|l|l|c|}
\hline & $\mathbf{2} \mathbf{~ m i n}$ & $\mathbf{6} \mathbf{m i n}$ & $\mathbf{1 0} \mathbf{~ m i n}$ & $\mathbf{2 0} \mathbf{~ m i n}$ & $\mathbf{3 0} \mathbf{m i n}$ \\
\hline MVC [\%] & $-2.81 \pm 5.39$ & $-2.32 \pm 4.19$ & $-2.87 \pm 5.31$ & $-9.26 \pm 9.67$ & $-17.01 \pm 13.09^{*}$ \\
\hline VA [\%] & $-1.81 \pm 3.14$ & $0.18 \pm 3.90$ & $-1.59 \pm 3.4$ & $-2.72 \pm 3.92$ & $-4.32 \pm 5.66$ \\
\hline MWVM [\%] & $-1.17 \pm 5.72$ & $-2.86 \pm 8.28$ & $-0.52 \pm 8.50$ & $-4.21 \pm 8.05$ & $-8.23 \pm 7.59$ \\
\hline MWVL [\%] & $4.07 \pm 10.33$ & $6.91 \pm 6.39$ & $5.07 \pm 8.43$ & $8.32 \pm 15.07$ & $6.9 \pm 28.92$ \\
\hline
\end{tabular}

Table 2. Changes in neuromuscular function over the time course of the slow component. MVC: maximal voluntary contraction, VA: voluntary activation, MWVM: M-wave amplitude of vastus medialis, and MWVL: M-wave amplitude of vastus lateralis. *Different from base line, $2 \mathrm{~min}, 6 \mathrm{~min}$, and $10 \mathrm{~min}(\mathrm{p}<0.05)$. Data are presented as mean $\pm \mathrm{SD}$.

Data and statistical analysis. Data were normalised by expressing the measures taken immediately after exercise as a percentage change relative to before exercise. This was completed to avoid day-to-day variations in measures that may occur. Normality test (Kolmogorov-Smirnov) and F-test of equality of variances were completed to test for normal distribution and equality of variance. One-way repeated measures analysis of variance (ANOVA) was used to test the effect of exercise duration on measurers of neuromuscular function. When a significant main effect was found, significant differences were located using Tukey's post hoc analysis test. Pearson correlation coefficient was used to assess relationships between the change of SCV\% and changes to neuromuscular parameters. Analyses were completed with Box and Tidwell tests, and the Theil method (Theil nonparametric regression technique). The Box and Tidwell test assesses whether the association between the slow component and fatigue is linear or not, and therefore related to time. In contrast, Theil's regression highlights, in a qualitative way, the points that are distant from the linear relationship. For all tests, significance was set at $\mathrm{p}<0.05$. Data are expressed as mean $\pm \mathrm{SD}$.

Ethical approval. The University of Auckland Human Participants Ethics Committee approved this study. Written informed consent was provided by all participants prior to participation. All procedures conformed to the latest revision (2013) of the Declaration of Helsinki.

\section{Results}

Oxygen uptake kinetics. Mean $\dot{\mathrm{VO}}_{2 \text { peak }}$ was $3.95 \pm 0.18 \mathrm{l} \cdot \mathrm{min}^{-1}$ and the mean power output corresponding to $30 \% \Delta$ was $200 \pm 11$ watts. During the three minutes of unloaded pedalling at $85 \mathrm{rpm}, \mathrm{V}_{2 \mathrm{~b}}$ reached a value of $0.85 \pm 0.191 \mathrm{~min}^{-1}$. Asymptotic amplitudes of the primary phase attained $1.85 \pm 0.381 \mathrm{~min}^{-1}$ with a time constant of $27.1 \pm 15.0 \mathrm{~s}$ and a time delay of $12.8 \pm 2.3 \mathrm{~s}$. Amplitude of the slow component at time 2,6,10,20, and $30 \mathrm{~min}-$ utes are presented in Table 1 . Values of SCV as a percentage of the primary component are also described.

Neuromuscular function. MVC measurement showed alteration over the course of exercise (Table 2). Post-hoc test revealed a significant reduction after 30 minutes of cycling compared to before exercise, 2, 6, and 10 minutes of exercise. After 20 minutes of exercise, a trend towards significance was observed compared to before exercise $(p=0.1)$ No effect of exercise duration was detected for VA (Table $2 ; p>0.05$ ).

No effect of exercise duration was detected for the M-wave amplitude of the VM and VL muscles (Table 2).

Twitch amplitude (Fig. 2A) showed a significant reduction at 30 minutes of exercise compared to before, 2 , 6 , and 10 minutes of exercise; $(p<0.05)$. A significant reduction was also observed after 20 minutes of exercise compared to before, 2 , and 6 minutes of exercise $(\mathrm{P}<0.05)$. Finally, significant differences were observed for 10 minutes of exercise compared to 2 and 6 minutes of exercise $(p<0.05)$.

P10 (Fig. 2B) and P100 (Fig. 2C) evolved in a similar manner. Specifically, significant differences were observed for 20 and 30 minutes compared to before, 2, 6, and 10 minutes of exercise $(\mathrm{p}<0.05)$. Furthermore, significant differences were observed at 10 minutes compared to 2 and 6 minutes $(p<0.05)$.

Significant differences for the P10/P100 ratio (Fig. 2D) were found for most exercise durations. A significantly lower P10/P100 ratio was observed at 30 minutes compared to before, 2, 6, and 10 minutes of exercise $(\mathrm{P}<0.05)$. After 20 minutes of exercise, differences were observed compared to before, 2 , and 6 minutes of exercise $(\mathrm{p}<0.05)$. Furthermore, significant differences were observed at 10 minutes compared to before and $2 \mathrm{~min}$ utes of exercise $(p<0.05)$. 

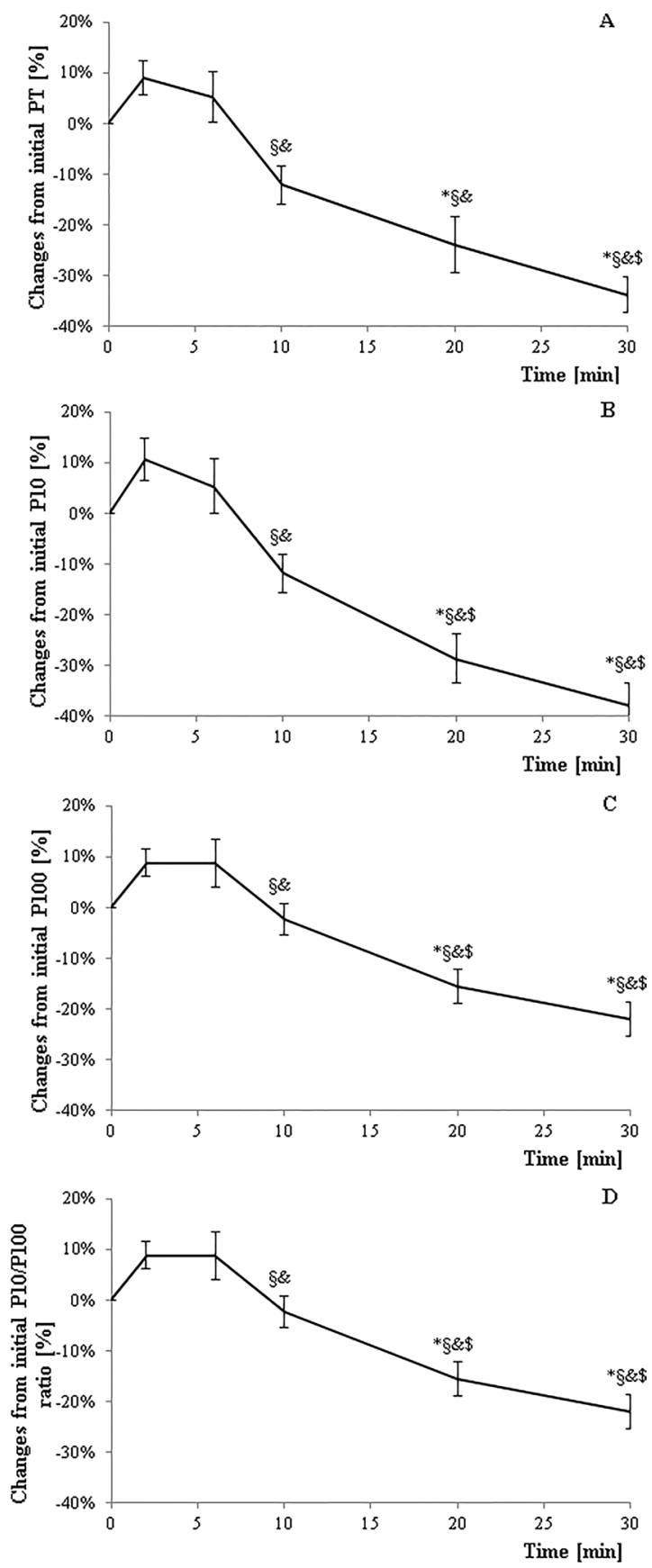

Figure 2. Neuromuscular alterations for peak twitch amplitude (A), $10 \mathrm{~Hz}$ paired (P10) stimulation (B), $100 \mathrm{~Hz}$ paired (P100) stimulation (C), and P10/P100 (D) over the course of exercise. *Significant difference from baseline $(\mathrm{p}<0.05)$; ${ }^{\S}$ Significant difference from 2 minutes. $(\mathrm{p}<0.05)$. ${ }^{\circledR}$ Significant difference from 6 minutes

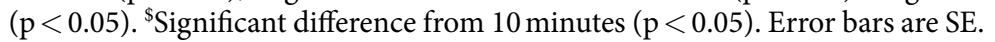

The SCV and fatigue. Correlation analysis was used to investigate relationships between the SCV\% and neuromuscular parameters (Table 3). Changes in M-wave amplitude for either of VL and VM, VA and MVC did not correlate with changes of the SCV relative to the primary phase. However, significant correlations were found between the SCV\% and PT, P10, P100 (tendency), and P10/P100 ratio. For these neuromuscular parameters, P10/ P100 showed the strongest correlation with SCV\% $\left(\mathrm{R}^{2}=0.88\right)$, followed by P10 $\left(\mathrm{R}^{2}=0.81\right), \mathrm{PT}\left(\mathrm{R}^{2}=0.81\right)$, and $\mathrm{P} 100$ ratio $\left(\mathrm{R}^{2}=0.72\right)$. In contrast, the Box and Tidwell's test was smaller than 0.05 (see Table 3 ) for correlation relationships suggesting that the relationship is non-linear and therefore unrelated over time. In addition, Theil's line (see Fig. 3) showed that during the first phase, only the slow component grew (the points of this phase are distant from Theil's line); while during the second phase, the slow component continued to grow but fatigue also grew (the points of this phase then line up with Theil's line). 


\begin{tabular}{|l|l|l|l|l|}
\hline \multirow{2}{*}{} & \multicolumn{2}{l|}{ Correlation } & \multicolumn{2}{l|}{ Box-Tidwell test } \\
\cline { 2 - 5 } & R & P & Z & P \\
\hline MWVM & -0.69 & 0.196 & -1.70 & 0.089 \\
\hline MWVL & 0.76 & 0.137 & -0.20 & 0.841 \\
\hline PT & -0.90 & 0.038 & -3.06 & 0.002 \\
\hline MVC & -0.72 & 0.172 & -5.57 & $<0.001$ \\
\hline VA & -0.52 & 0.370 & -5.48 & $<0.001$ \\
\hline P10 & -0.90 & 0.036 & -3.39 & $<0.001$ \\
\hline P100 & -0.85 & 0.065 & -3.97 & $<0.001$ \\
\hline P10/P100 & -0.94 & 0.019 & -4.50 & $<0.001$ \\
\hline
\end{tabular}

Table 3. Correlation coefficient and Box-Tidwell test between the slow component amplitude, as a percentage of the primary phase, and neuromuscular function. MWVM: M-wave amplitude of vastus medialis, MWVL: M-wave amplitude of vastus lateralis, PT: Peak Torque of the single twitch, MVC: maximal voluntary contraction, VA: voluntary activation, P10: peak torque at $10 \mathrm{~Hz}$ doublet stimulation, P100: peak torque at $100 \mathrm{~Hz}$ doublet stimulation, P10/P100: ratio of peak torque between $10 \mathrm{hz}$ and $100 \mathrm{hz}$ doublet stimulation, R: correlation coefficient, $\mathrm{Z}$ score statistic, P: significance.

\section{Discussion}

The main finding from the present study was that alterations to force production by the knee extensor muscles were present during exercise at an intensity of $30 \% \Delta$, which correlated with the development of the SCV, however, a temporal relationship between the development of the SCV and fatigue does not appear to exist.

Origin of fatigue observed during exercise. With neuromuscular fatigue defined as a reduction in force generating capacity ${ }^{28}$, loss of MVC torque is used as a general index for evaluating the extent of neuromuscular fatigue. In the present study, MVC torque, compared to the beginning of exercise, was found to be significantly reduced only after 30 minutes of cycling at $30 \% \Delta$. However, while loss of MVC torque is a general index of fatigue, it does not provide information regarding the site of alterations (i.e. neuromuscular fatigue etiology). To determine the origin of the neuromuscular fatigue caused by various durations of cycling at $30 \% \Delta$, electrical stimulations were delivered at rest, as well as during MVC, allowing for the evaluation of VA, action potential transmission and propagation, and neuromuscular properties. VA, which is commonly used to evaluate central fatigue $^{29}$, was not significantly affected by any exercise durations in the present study. The absence of significant central fatigue suggests that declines in motivation, afferent feedback, or central drive were not present, or that declines in central drive was countered by increased motivation ${ }^{10,30}$. It subsequently suggests a peripheral origin for the induced neuromuscular fatigue. Muscle membrane excitability and neuromuscular propagation appeared to be well preserved, as highlighted by the lack of alterations in VL and VM M-wave amplitudes. In contrast, reductions in evoked forces suggests the presence of peripheral fatigue. Interestingly, signs of peripheral fatigue were observed following shorter exercise durations, suggesting that evoked forces might be more sensitive than MVC for detecting fatigue when it is of peripheral origin. Indeed, PT, P10 and P100 were already reduced after 20 minutes of cycling compared to the beginning of exercise. As M-wave amplitudes were unaltered at all-time points, reductions in evoked forces can highlight either alterations in sarcoplasmic reticulum $\mathrm{Ca}^{2+}$ handling $^{8}$ or alterations occurring at the cross-bridge level such as reduced myofibrillar $\mathrm{Ca}^{2+}$ sensitivity, and/or reduced capacity for cross-bridge to produce force ${ }^{31,32}$. Further supporting excitation-contraction failure, the P10/P100 ratio was found to be reduced following 10,20, and 30 minutes of exercise compared to the start of exercise suggesting the presence of low frequency fatigue ${ }^{33}$. A study completed on rat gastrocnemius muscle ascribed low-frequency fatigue to $\mathrm{Ca}^{2+}$ handling alterations rather than to processes occurring at the cross-bridge level ${ }^{34}$. Indeed, altered $\mathrm{Ca}^{2+}$ handling is believed to occur with $\mathrm{P}_{\mathrm{i}}$ accumulation during the development of fatigue and its subsequent precipitation with $\mathrm{Ca}^{2+}$ within the sarcoplasmic reticulum ${ }^{35}$. However, the exact mechanisms responsible for low-frequency fatigue remain unclear as previous results, also obtained using rodents, showed that the site (i.e. $\mathrm{Ca}^{2+}$ handling $v$ s. cross-bridge level) responsible for this low-frequency fatigue is dependent on the antioxidant status of the individual ${ }^{36}$. Therefore, based on the measures in the present study, it is likely that the observed neuromuscular fatigue following 20 and 30 minutes of cycling at $30 \% \Delta$ is a result of peripheral rather than central fatigue. Based on the literature, while speculative, it suggests that fatigue it is from either impaired Ca handling or reduced cross-bridge kinetics. ${ }^{31,32}$.

The SCV and fatigue. Significant correlations were found between the SCV\% and PT, P10, P100, and P10/ $\mathrm{P} 100$. This finding is supportive of the theory regarding the presence of fatigue required to elicit the SCV. In contrast, for these parameters, the Box and Tidwell's test showed that the relationship between the development of the SCV and the alterations of the neuromuscular properties of knee extensor muscles were non-linear and therefore unrelated over time. In addition, Theil's line (see Fig. 3) showed two distinct phases; the first phase where only the slow component grew (the points of this phase are away from Theil's line); while during the second phase, the slow component continued to grow but fatigue also grew (the points of this phase then line up with Theil's line). (see Fig. 3). In other words, the development of the SCV, in fact, occurred mainly between 2-10 minutes during which neuromuscular properties were relatively stable (only a reduction in the P10/100 ratio was observed after 10 minutes of cycling). In contrast, PT, P10 and P100 were significantly reduced only after 20-30 minutes 

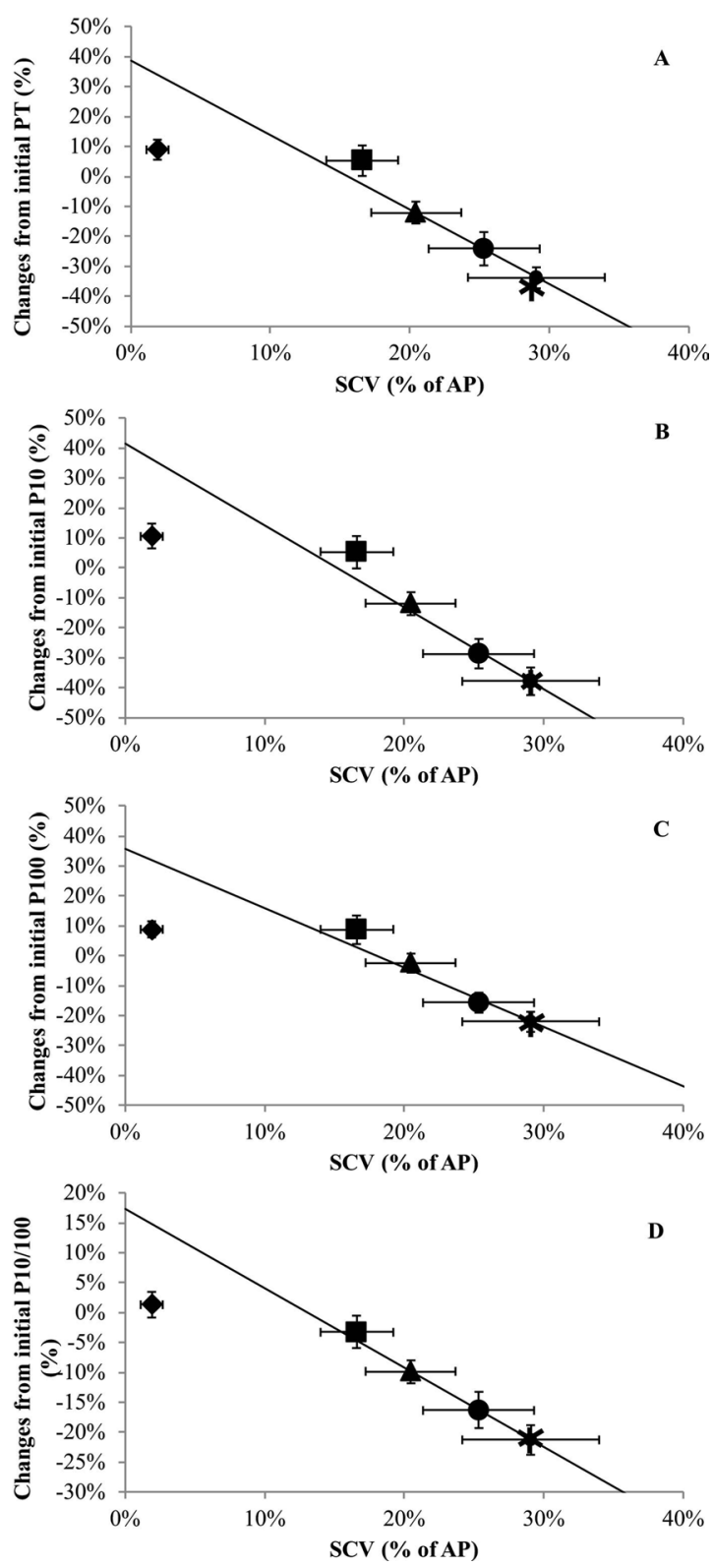

Figure 3. The relationship between peak twitch amplitude (A), $10 \mathrm{~Hz}$ paired (P10) stimulation (B), $100 \mathrm{~Hz}$ paired (P100) stimulation (C), and P10/P100 (D) and the change in SCV relative to the primary phase. 2 minutes; 6 minutes; 10 minutes; 20 minutes; and * 30 minutes represent average values. Theil's line is characterised by the dashed line. Error bars are SE. Error bars in the figures are presented as SE for more clarity.

of exercise compared to baseline values. These results suggest that the development of fatigue due to alterations of neuromuscular properties is not an essential requirement to elicit the SCV at least during the first 10 minutes of exercises. This finding is in line with those from Thistlethwaite and colleagues ${ }^{37}$. They showed that, during heavy cycling exercise, when preceded either by heavy exercise or by heavy knee extensions (requiring twofold greater muscle activation relative to heavy exercise), $\tau_{\mathrm{p}}$, gain of the primary response, and the amplitude of the SCV were similar between protocols. The authors concluded that muscle fatigue is not a determining factor for the development of the SCV. Hopker and colleagues attested similar results. Participants completed either a non-metabolically stressful 100 intermittent drop-jumps protocol (pre-fatigue condition) or rest (control) for 33 minutes. The results of their study showed that locomotor muscle fatigue, tested by the reduction in power in the maximal voluntary cycling power test, was not associated with the development of the $\mathrm{SCV}^{38}$. Interestingly, the magnitude of the SCV was not significantly different between the two conditions despite significant differences in locomotor muscle fatigue. Recently, Dos Nascimento Salvador and colleagues published a study looking at the cause-effect relationship between the SCV and fatigue. They switched from constant work rate to isokinetic pedaling to quantify reductions in peak torque at three and eight minutes, with and without priming exercise. Results showed that the SCV after priming was reduced but there were no significant differences between 
conditions regarding the magnitude of the reduction of maximal isokinetic force and power at three and eight minutes ${ }^{39}$. This observation refutes a cause-effect relationship between fatigue and the development of the SCV.

However, the findings from this study are in contrast with the results by Keir and colleagues. Correlations were shown in both studies between some measures of fatigue and the SCV, however, a temporal association was only found in one study ${ }^{9}$. In one perspective, this difference highlights the importance of exercise intensity. Indeed, in the present study, step transition exercise was in the heavy domain, while the study by Keir and colleagues was in the severe domain ${ }^{9}$. In addition, the amplitude and type of fatigue was potentially different, as assessed by the difference in reduction of MVC after 18-20 minutes ( $9 \%$ for the present study vs $22 \%$ in the study by Keir and colleagues). If the SCV is related to fatigue parameters, it should be present in both exercise intensity domains. However, this was not the case, which suggests that the SCV may not be related to fatigue parameters.

The results in the present study are in agreement with results from a previous study regarding changes to velocity-specific peak power during cycling. Cannon et al. ${ }^{17}$ observed a reduction in velocity-specific peak power, which correlated with the SCV. However, as was observed in the present study, the reduction they observed was not temporally related to the development of the SCV. The reduction in velocity-specific peak power occurred prior to the SCV in their study, while excitation-contraction coupling was altered after the development of SCV in the present study. Nevertheless, both reported no changes during the development of the SCV which suggests that those alterations are likely not essential for the development of the SCV. If alterations to neuromuscular properties are not involved during the development of the SCV, at least during exercise in the heavy domain, it may be possible that the $\dot{\mathrm{V}} \mathrm{O}_{2}$ cost of force production may increase within a given fiber population. A progressive inhibition of ATP supply by anaerobic glycolysis, an increase in ATP usage per power output, and/or a reduction of ATP production per mole of oxygen $\left(\mathrm{P} / \mathrm{O}_{2}\right.$ ratio) are probably implicated in the $\mathrm{SCV}^{40}$. However, the documentation of a cause-effect relationship during exercise between muscle fatigue and reduced efficiency remains unknown.

Experimental consideration. As with the study by Keir and colleagues (2016), at the end of exercise, the time to transfer the subject from the ergometer to the Biodex before the start of neuromuscular testing was less than one minute. One could argue that fatigue was already modified, and consequently the interpretation of the data in relation to fatigue during exercise is limited. Simply, fatigue is likely to have been underestimated in the present study and the measurement of fatigue during exercise would have been more appropriate. However, neuromuscular measurements were taken after a similar amount of time after each exercise, for each participant, and consequently, the change of the robustness of the relationship between fatigue and the SCV is likely to have been marginal, which should not change the general conclusions of the present study. Furthermore, the cause (fatigue) has to precede the effect (SCV); however, the data from the present study indicates that this was not the case. A further limitation is the fact that fatigue was measured during static contractions whereas cycling is a dynamic movement.

\section{Conclusion}

Fatigue in the present study was observed during exercise completed at $30 \% \Delta$ and which was at least 20 minutes in duration. Indirectly, these results suggest that the observed fatigue appears to be a result of impaired $\mathrm{Ca}^{2+}$ handling and/or reduced capability of cross-bridges to produce force. While significant correlations between the SCV relative to the primary phase and neuromuscular parameters were found, a temporal relationship between the development of the SCV and fatigue does not appear to exist. Therefore, it would seem that the alteration of neuromuscular properties in muscle is not required for the development of the SCV.

\section{Data availability}

The datasets generated and analysed during the current study are available from the corresponding author on reasonable request.

Received: 20 December 2019; Accepted: 13 April 2020;

Published online: 07 May 2020

\section{References}

1. Grassi, B. Oxygen uptake kinetics: old and recent lessons from experiments on isolated muscle in situ. European journal of applied physiology 90, 242-249, https://doi.org/10.1007/s00421-003-0994-0 (2003).

2. Hughson, R. L. Oxygen uptake kinetics: historical perspective and future directions. Applied Physiology Nutrition and MetabolismPhysiologie Appliquee Nutrition Et Metabolisme 34, 840-850, https://doi.org/10.1139/h09-088 (2009).

3. Jones, A. M. \& Poole, D. C. In Oxygen Uptake Kinetics in Sport, Exercise and Medicine (eds. Jones, A. M. \& Poole, D. C.) 3-35 (Routledge, 2005).

4. Xu, F. \& Rhodes, E. C. Oxygen uptake kinetics during exercise. Sports Medicine 27, 313-327 (1999).

5. Hansen, J. E., Sue, D. Y., Oren, A. \& Wasserman, K. Relation of oxygen uptake to work rate in normal men and men with circulatory disorders. The American journal of cardiology 59, 669-674 (1987).

6. Whipp, B. J. \& Wasserman, K. Oxygen uptake kinetics for various intensities of constant-load work. Journal of Applied Physiology 33, 351-356 (1972).

7. Poole, D. C. et al. Contribution of excising legs to the slow component of oxygen uptake kinetics in humans. J Appl Physiol 71, 1245-1260 (1991).

8. Allen, D. G., Lamb, G. D. \& Westerblad, H. Skeletal Muscle Fatigue: Cellular Mechanisms. Physiological Reviews 88, 287-332, https:// doi.org/10.1152/physrev.00015.2007 (2008).

9. Keir, D. A. et al. The slow component of pulmonary $\mathrm{O} 2$ uptake accompanies peripheral muscle fatigue during high-intensity exercise. Journal of Applied Physiology 121, 493-502, https://doi.org/10.1152/japplphysiol.00249.2016 (2016).

10. Ament, W. \& Verkerke, G. J. Exercise and Fatigue. Sports Medicine 39, 389-422 (2009).

11. Cannon, D. T. et al. Skeletal muscle ATP turnover by $31 \mathrm{P}$ magnetic resonance spectroscopy during moderate and heavy bilateral knee extension. J Physiol 592, 5287-5300, https://doi.org/10.1113/jphysiol.2014.279174 (2014). 
12. Grassi, B., Rossiter, H. B. \& Zoladz, J. A. Skeletal muscle fatigue and decreased efficiency: two sides of the same coin? Exerc Sport Sci Rev 43, 75-83, https://doi.org/10.1249/JES.0000000000000043 (2015).

13. Poole, D. C. \& Jones, A. M. Oxygen uptake kinetics. Comprehensive Physiology 2, 933-996, https://doi.org/10.1002/cphy.c100072 (2012).

14. Rossiter, H. B. Exercise: Kinetic considerations for gas exchange. Comprehensive Physiology 1, 203-244, https://doi.org/10.1002/ cphy.c090010 (2011).

15. Decorte, N., Lafaix, P. A., Millet, G. Y., Wuyam, B. \& Verges, S. Central and peripheral fatigue kinetics during exhaustive constantload cycling. Scandinavian Journal of Medicine \& Science in Sports, 381-391, https://doi.org/10.1111/j.1600-0838.2010.01167.x (2010).

16. Keir, D. A. et al. The slow component of pulmonary $\mathrm{O} 2$ uptake accompanies peripheral muscle fatigue during high-intensity exercise. Journal of applied physiology (Bethesda, Md.: 1985) 121, 493-502, https://doi.org/10.1152/japplphysiol.00249.2016 (2016).

17. Cannon, D. T., White, A. C., Andriano, M. F., Kolkhorst, F. W. \& Rossiter, H. B. Skeletal muscle fatigue precedes the slow component of oxygen uptake kinetics during exercise in humans. The Journal of physiology 589, 727-739, https://doi.org/10.1113/ jphysiol.2010.197723 (2011).

18. Froyd, C., Millet, G. Y. \& Noakes, T. D. The development of peripheral fatigue and short-term recovery during self-paced highintensity exercise. J Physiol 591, 1339-1346, https://doi.org/10.1113/jphysiol.2012.245316 (2013).

19. Whipp, B. J., Davis, J. A., Torres, F. \& Wasserman, K. A test to determine parameters of aerobic function during exercise. Journal of Applied Physiology 50, 217-221 (1981).

20. Bailey, S. J. et al. Inspiratory muscle training enhances pulmonary O-2 uptake kinetics and high-intensity exercise tolerance in humans. Journal of Applied Physiology 109, 457-468 (2010).

21. Weissman, M. L. et al. Cardiac output increase and gas exchange at start of exercise. Journal of Applied Physiology 52, 236-244 (1982).

22. Paterson, D. H. \& Whipp, B. J. Asymmetries of oxygen uptake transients at the on- and offset of heavy exercise in humans. Journal of Physiology-London 443, 575-586 (1991).

23. Whipp, B. J., Ward, S. A., Lamarra, N., Davis, J. A. \& Wasserman, K. Parameters of ventilatory and gas exchange dynamics during exercise. Journal of Applied Physiology 52, 1506-1513 (1982).

24. Rossiter, H. B. et al. Effects of prior exercise on oxygen uptake and phosphocreatine kinetics during high-intensity knee-extension exercise in humans. The Journal of physiology 537, 291-303, https://doi.org/10.1111/j.1469-7793.2001.0291k.x (2001).

25. Murgatroyd, S. R., Ferguson, C., Ward, S. A., Whipp, B. J. \& Rossiter, H. B. Pulmonary O2 uptake kinetics as a determinant of highintensity exercise tolerance in humans. Journal of applied physiology 110, 1598-1606 (2011).

26. Merton, P. A. Voluntary strength and fatigue. Journal of Physiology-London 123, 553-564 (1954).

27. Strojnik, V. \& Komi, P. V. Neuromuscular fatigue after maximal stretch-shortening cycle exercise. Journal of Applied Physiology 84, 344-350 (1998).

28. Gandevia, S. C. Spinal and Supraspinal Factors in Human Muscle Fatigue. Physiological Reviews 81, 1725-1789 (2001).

29. Vollestad, N. K. Measurement of human muscle fatigue. Journal of Neuroscience Methods 74, 219-227 (1997).

30. Bigland-Ritchie, B., Dawson, N. J., Johansson, R. S. \& Lippold, O. C. J. Reflex origin for the slowing of motoneurone firing rates in fatigue of human voluntary contractions. Journal of Physiology-London 379, 451-459 (1986).

31. Metzger, J. M. \& Moss, R. L. Effects of tension and stiffness due to reduced pH in mammalian fast- and slow-twitch skinned skeletal muscle fibres. The Journal of Physiology 428, 737-750 (1990).

32. Place, N., Yamada, T., Bruton, J. D. \& Westerblad, H. Muscle fatigue: from observations in humans to underlying mechanisms studied in intact single muscle fibres. European journal of applied physiology 110, 1-15, https://doi.org/10.1007/s00421-010-1480-0 (2010).

33. Jones, D. A. High- and low-frequency fatigue revisited. Acta Physiologica Scandinavica 156, 265-270 (1996).

34. Watanabe, D. et al. Contribution of impaired myofibril and ryanodine receptor function to prolonged low-frequency force depression after in situ stimulation in rat skeletal muscle. J Muscle Res Cell Motil 36, 275-286, https://doi.org/10.1007/s10974-0159409-1 (2015).

35. Westerblad, H. \& Allen, D. The effects of intracellular injections of phosphate on intracellular calcium and force in single fibres of mouse skeletal muscle. Pflügers Archiv European Journal of Physiology 431, 964-970, https://doi.org/10.1007/s004240050092 (1996).

36. Bruton, J. D. et al. Reactive oxygen species and fatigue-induced prolonged low-frequency force depression in skeletal muscle fibres of rats, mice and SOD2 overexpressing mice. J Physiol 586, 175-184, https://doi.org/10.1113/jphysiol.2007.147470 (2008).

37. Thistlethwaite, J. R., Thompson, B. C., Gonzales, J. U. \& Scheuermann, B. W. Prior heavy knee extension exercise does not affect $\$ \$$ $\operatorname{dot}\{\mathrm{V}\} \backslash \mathrm{hbox}\{\mathrm{O}\}_{-}\{2\} \$ \$$ kinetics during subsequent heavy cycling exercise. European Journal of Applied Physiology 102, 481-491, https://doi.org/10.1007/s00421-007-0614-5 (2008).

38. Hopker, J. G., Caporaso, G., Azzalin, A., Carpenter, R. \& Marcora, S. M. Locomotor Muscle Fatigue Does Not Alter Oxygen Uptake Kinetics during High-Intensity Exercise. Frontiers in Physiology 7, https://doi.org/10.3389/fphys.2016.00463 (2016).

39. do Nascimento Salvador, P. C., Souza, K. Md, De Lucas, R. D., Guglielmo, L. G. A. \& Denadai, B. S. The effects of priming exercise on the V O2 slow component and the time-course of muscle fatigue during very-heavy-intensity exercise in humans. Appl Physiol Nutr Metab 43, 909-919, https://doi.org/10.1139/apnm-2017-0769 (2018).

40. Korzeniewski, B. \& Zoladz, J. A. Possible mechanisms underlying slow component of V'O2 on-kinetics in skeletal muscle. Journal of Applied Physiology, 1240-1249, https://doi.org/10.1152/japplphysiol.00027.2015 (2015).

\section{Acknowledgements}

The authors would like to thank all participants who took part in this study and all technical members at The Department of Sport and Exercise Science, Faculty of Science, University of Auckland, Auckland, New Zealand.

\section{Author contributions}

F.B. conceived and designed the experiments. Data collection was completed by F.B. and T.G. F.B., T.G., S.C.A., J.R. and J.A. analysed, interpreted, revisited, and approved the final version of the manuscript.

\section{Competing interests}

The authors declare no competing interests.

\section{Additional information}

Correspondence and requests for materials should be addressed to S.C.

Reprints and permissions information is available at www.nature.com/reprints.

Publisher's note Springer Nature remains neutral with regard to jurisdictional claims in published maps and institutional affiliations. 
(c) (i) Open Access This article is licensed under a Creative Commons Attribution 4.0 International License, which permits use, sharing, adaptation, distribution and reproduction in any medium or format, as long as you give appropriate credit to the original author(s) and the source, provide a link to the Creative Commons license, and indicate if changes were made. The images or other third party material in this article are included in the article's Creative Commons license, unless indicated otherwise in a credit line to the material. If material is not included in the article's Creative Commons license and your intended use is not permitted by statutory regulation or exceeds the permitted use, you will need to obtain permission directly from the copyright holder. To view a copy of this license, visit http://creativecommons.org/licenses/by/4.0/.

(c) The Author(s) 2020 